\title{
Toxoplasmose canine en République Centrafricaine
}

\author{
par A. GODARD
}

Il nous a paru intéressant de signaler 'existence de la toxoplasmose canine en République Centrafricaine, ainsi que nous l'avons constatée plusieurs fois à la clinique journalière du Service à Bambari. A la lecture d'un article «Co'loque sur les toxoplasmes et les toxop'asmoses animales » par le Professeur Guithon de l'Ecoie Vétérinaire d'Alfort (*), nous devors d'avoir pu orienter notre diagnostic. Celui-ci nous a été confirmé par A. Provost du Laboratoire de Farcha-Fort-Lamy.

L'affection semble toucher principa'ement les chiens d'origine métropolitaine. Jusqu'à prèsent, nous ne l'avons pas décelée sur les chiens inaigènes. Sans doute, ceux-ci sont également infestés, mais la maladie ne se manifeste pas ce manière aussi brutale. Plusieurs cas, décrios pius loin, se rapportent à des arimaux se méme souche. Il s'agit d'une chienne berger a iemand. morte deux mois après la mise-oas, à sâ troisième portée. Parmi tous ses produits, qui furent distribués en différents endroits du territcire, peu ont survécu. A Bamoari, nous avons examiré la chienne et cleux descerajan-s de portées différentes. Le -oxopiasme a été icentifié sur un des chiens, dort nous avons eu la posilio ité de pratiquer l'autopsie moins de deux reures apès la mort.

Instruit mainterant par "excór érce, nois faisons un rapprochement avec d'autres chiens. qui présentés antérieurement à a consultarion. nous ont obligé d'avouer sotre incompráhersion. Bien que res observations soiert sommaires,

Reçu pour pubiication : ivi let 1959.

(*) Rec. Méd. vét., 1957, 133, 715. nous avons jugé utile d'en parler. Nous pensons qu ${ }^{\prime \prime .}$ sagissait dans tous ces cas de toxoplasmose.

\section{OBSERVATIONS CLINIQUES}

Observation I. Une chienne berger est en traitement. Après ses chaleurs, elle continue à préserter dej pertes. Mais elle garde bon appétit et he parait pas autrement malade.

Queques jours plus tard, le propriétaire nous apporte sa chienne morte pandant la nuit. L'anima a fai- Lne crise nerveuse épileptiforme au terme de aquelle il a succombé. L'autopsie révèle des lésions congestives ef nous concluons ذे lempoisonnement.

Observation II. Une chienne est amenée le ira-ir à a consuitation en décembre 1958. Elle tréóigne sans arrêt de ses pattés antérieures et se mainier- difficilement debout. Le soir, la bóse est trouvée morte.

L'autopsie est faite rapidement, car il fait céià juit. De couveau, nous concluons à l'emcolsonemert.

Observation III. Ure chienne croisée berger a."emand âgée de trois ans, est présentée à la consutation début janvier. Elle a mis bas delx mo's auparavant el rourri quatre chiots sevrés depuis trois semaines. Depuis qu'elle a cessé "al"aitement, elie a maigri considérablement. Elle refuse maintenant toute nourriture, accepte seu'ement de boire un peu d'eau.

On note une conjonctivite suppurée, les yeux enfoncés dars l'orbite. La salivation est abondante 'sa'ive mousseusel, la langue pendante. Les 
membres antérieurs ont des contractions cloniques. La bôte roste couchée, très abattue.

Pensant encore à l'empoisonnement, nous faisons du chloral en intra-péritonéal. Le lendemain, it se produit une légère amélioration. Les jours suivants, au contraire, les symptômes s'aggravent. L'animal fait des efforts de vomissement. La respiration est devenue de plus en plus pénible. Au quatrième jour, on voit apparâitre des pétéchies sur la peau du ventre. II meurt pendant la nuit.

L'autopsie pratiquée six heures après la mort montre une congestion de tous les organes. On remarque un foyer de pneumonie lobaire, de la congestion hépatique, une rate légèrement hypertrophiée, molle ef grisâtre, également sur l'intestin une entérite assez vive.

Examens pratiqués : frottis de sang et de foie.

Nous pensons qu'il s'agit d'une affection que nous ignorons et peut-être la toxoplasmose. Mais l'autopsie était tardive et le résultat de l'examen des frottis par le Laboratoire de Farcha est négatif.

Observation IV. Un des produits de la chienne précédente a d'abord eu la patte arrière gauche fracturée par un camion. La fracture s'est d'ailleurs mal réduite, car le propriétaire a coupé le plâtre trop tôt. II est'ramené à la visite début février.

En quelques jours, il a beaucoup maigri, ne mange plus, accepte seulement l'eau. Nous notons une température de $39^{\circ}$, conjonctivite, les pupilles dilatées. L'animal paraît hébété. La respiration est pompante, le choc précordial accentué, le ventre douloureux.

Nous essayons, comme traitement un sulfone per os.

Le soir, le chien s'évade et il est retrouvé le lendemain matin par une personne qui vient nous avertir de la présence d'un chien suspect dans sa concession. Le propriétaire ramène le chien chez lui où il meurt deux jours plus tard.

L'autopsie, faite deux heures après la mort, montre d'importantes lésions pulmonaires. Un lobo ost hépatisć of sur le poumon droit surtout, sont disséminées des taches hémorragiques ef en relief de la grosseur d'un grain de mil à une cerise. Le foie est congestionné, la rate est bleuâtre nettement hypertrophiée. Le rein est atteint de néphrite aigü̈ et la vessie est pleine d'une urine jaune foncée.

Examens pratiqués : nous avons fait différents frottis de corne d'Ammon, cortex cérébral, foie, poumon et sang colorés au May-Grünwald ef Giemsa, qui sont envoyés au Laboratoire de Farcha; la réponse est formelle : elle indique la présence de toxoplasmes dans le foie et le cerveau.

Observation V. Un propriétaire amène son chien qui ne mange plus. Nous prenons la température, $39^{\circ} 7$. Les muqueuses oculaires sont tròs congestionnées. L'animal a une salivation mousseuse, sa démarche est ébrieuse. Les cas antérieurs nous ayant fait supposer la toxoplas. mose, nous prescrivons un sulfone per os à la dose de $10 \mathrm{mg} / \mathrm{kg}$. Trois jours après, la bête va mieux et à la fin du traitement de huit jours, est complètement guérie.

Observation VI. Un chien croisé épagneul âgé de deux ans est présenté le 24 avril 1959 par le propriétaire qui a déià perdu une chienne lobservation II). Cet animal a fait une fugue de neuf jours. De retour chez son maitre, il accuse une maigreur prononcée. Son appétit est presque nul.

A l'examen clinique, nous notons une tempéráture de $39^{\circ}$, les conjonctives sont violacées avec une suppuration au coin de l'œil, le nez souillé par un jetage purulent.

Nous faisons un étalement de sang qui se révèle négatif. Nous craignons une rechute de piroplasmose sur ce chien traité au zothélone un mois avant.

Nous rassurons le propriétaire, en disant que le chien a dû suivre une chienne et nous conseillons comme traitement : auréomycine, gouttes dans le nez et les yeux.

Le chien est ramené mort le 30 avril. La veille. le propriétaire nous avait signalé que son chien était mourant.

L'autopsie, faite trois heures après la mort, montre un chien très amaigri, les yeux sont enfoncés dans. l'orbite. Les lésions pulmonaires sont accusćes. La pneumonie est bilatérale, l'hépatisation est totale au niveau des lobes intermédiaires, où se distinguent des petits foyers de nécrose isolés ou en amas de la grosseur d'une 
tête d'épingle à une lentille, durs à la palpation et faisant saillie à la surface de l'organe. La rate est hypertrophiée $(2$ à 3 fois son volume normal), et sur un côté, on remarque une tache triangulaire telle une adhérence. On constate également une néphrite aiguë et dans la vessie, une urine jaune foncée.

Examens pratiqués : prélèvements du cerveau dans le formol à 10 p. 100, frottis de foie et de rate envoyés au Laboratoire de Farcha. Ceíli-ci nous confirme la « présence de nombreux toxoplasmes dans les frottis de foie et de rate $»$.

Observation VII. Un chien berger âgé de un an est vacciné contre la rage (vaccin Flury du Laboratoire de Farcha). Trois semaines se passent. Le propriétaire nous ramène le chien qui depuis plusieurs jours ne mange plus, a maigri sensiblement ef manifeste des troubles psychiques. Par exemple, il saute sur le lit de son maître, ce qu'il n'a jamais fait, ou il se cache sous une armoire. Par moments, le chien sembie fou. II présente en outre, une conjonctivite granuleuse avec suppuration au coin de l'œil.

Nous prescrivons des comprimés de sulfones $\left({ }^{*}\right)$ pendant une semaine. Le chien revu huit jours plus tard, a repris appétit, le poil est brillant. la conjonctivite a disparu. II ne manifeste pius aucun trouble.

\section{CONCLUSIONS}

Si la toxoplasmose canine en République Centrafricaine est désormais un fait acquis, ies aspects cliniques de cette affection restent difficiles à interpréter. Souvent, la maladie fait suite à un état physiologique ou pathologique ayant diminué la résistance du sujet. Chez la chienne, nous la voyons apparaitre après les chaleurs ou la mise-bas, chez le chien, comme suite de fracture ou de piroplasmose, ou même comme une conséquence de la vaccination antirabique.

Selon nos observations, on peut distinguer deux formes d'évolution :

1" Les cas aigus ou suraigus qui durent quelques heures à une journée au maximum. Ici, le tabeau rappelle la crise d'épilepsie ou l'empoisonnement:

$2^{\prime \prime}$ Les formes plus lentes où le début de la maladie est insidieux. Elles se signalent par un amaigrissement rapide du sujet et s'accompagnent toujours de conjonctivite purulente. L'inappétence est la règle. Les signes, nerveux sont atténués mais rarement absents. Ce sont des troubles psychiques caractérisés par une fugue ou des modifications passagères du comportement. Ils inquiètent parfois le proprićtaire à tel point qu'il craint que son animal soit enragé. Dans d'autres cas, les troubles sont locomoteurs et se signalent par de l'ataxie ou des contractures.

La température est peu élevée.

L'état général du malade baisse. Il a du mal à se tenir debout. Il finit par se coucher définitivement. A cette période, les yeux sont enfoncés dans l'orbite, la conjonctive est violacée, la bouche est souillée d'une bave mousseuse, la langue pendante. Les signes pulmonaires sont accusés. L'animal meurt dans un état cachectique après une agonie de plusieurs jours qui ressemble à la phase terminale de la maladie de Carré.

Ainsi, la toxoplasmose s'ajoute aux autres maladies à manifestations nerveuses qui, en milieu tropical surtout, permettent une confusion possible avec la rage.

(*) Le diaminodiphénylsulfone. comprimés à $100 \mathrm{mg}$, utilisé en médecine humaine contre la lèpre rous a été fourni par le Service de Santé. 


\section{SUMMARY}

\section{Canine toxoplasmosis in the Republic of Central Africa.}

Though canine toxoplasmosis in Republique Centrafricaine is well-recognised, the clinical symptoms of this condition remain difficult to diagnose and may be confused with those of rabies. Often the disease follows physiological or pathological events (i.e. heat period whelping, rabies inoculation, etc.). The author describes several cases in dogs, which have been ascribed to toxoplasmosis or diagnosed as toxoplasmosis after laboratory examination. In the acute form, death occurs within one day with symptoms reminiscent of poisoning or epilepsy. In the chronic form, rapid emaciation, purulent conjunctivitis and mild nervous symptoms (psychical and locomotor) can be seen. Death occurs after several days of agony.

\section{RESUMEN}

\section{Toxoplasmosis canina en República Centroafricana.}

Si la toxoplasmosis canina en República Centroafricana es por demás un hecho sabido, los aspectos clínicos de esta afección permanecen dificiles a la interpretación y pueden prestarse a confusión con la rabia. Corrientemente la enfermedad sigue a un estado fisiológico a patológico (calores, partos: vacunación antirrábica; afecciones diversas). El autor cita un gran número de observaciones concernientes a perros cuya enfermedad ha sido clasificada como una toxoplasmosis - bien ha sido reconocida por los exámenes de laboratorio como una verdadera toxoplasmosis. En la forma aguda la muerte sobreviene en menos de un dia con sintomas que recuerdan un envenenamiento o una crisis de epilepșia. En la forma crónica se aprecia un adelgazamiento rápido que conduce a la caquexia, una conjuntivitis purulenta, signos nerviosos leves (trástornos psiquicos y de locomoción). La muerte sobreviene despues de una agonía que dura varios dias. 